\title{
Interleukin-6 gene variants are associated with reduced risk of chronicity in hepatitis B virus infection in a Malaysian population
}

\author{
BEHNAZ RIAZALHOSSEINI $^{1}$, ZAHURIN MOHAMED ${ }^{1}$, \\ YAMUNAH DEVI APALASAMY ${ }^{2}$, NOOR SHAFILA SHAFIE ${ }^{1}$ and ROSMAWATI MOHAMED ${ }^{3}$ \\ ${ }^{1}$ Department of Pharmacology, Faculty of Medicine; ${ }^{2}$ Social Wellbeing Research Centre, \\ Faculty of Economics and Administration; ${ }^{3}$ Department of Medicine, Faculty of Medicine, \\ University of Malaya, Kuala Lumpur 50603, Malaysia
}

Received February 7, 2018; Accepted June 29, 2018

DOI: $10.3892 /$ br.2018.1126

\begin{abstract}
Interleukin-6 (IL-6) is a cytokine with a critical role in regulating the immune response to infectious disease. Studies have indicated that polymorphisms in the $I L-6$ gene may be linked to hepatitis B virus (HBV) infection. The purpose of the present study was to examine the association among IL-6 SNPs and haplotypes with HBV infection risk in a Malaysian population. A total of 1,246 Malaysian subjects with and without chronic hepatitis B were recruited for this study. Three $I L-6$ polymorphisms (rs2069837, rs1800796 and rs2066992) were genotyped using a Sequenom MassARRAY ${ }^{\circledR}$ platform. The results suggested that GC and CC genotypes of rs1800796 as well as GT and TT genotypes of rs2066992 were associated with protection against HBV infection $(\mathrm{P}<0.001)$. Furthermore, haplotypes GG and CT exhibited a significant association with protection against $\mathrm{HBV}(\mathrm{P}=0.003$ and $=0.005$, respectively); and haplotypes $\mathrm{GG}$ and $\mathrm{CT}$ exhibited a significant association with clearance of HBV infection $(\mathrm{P}=0.035$ and $=0.037$, respectively). The present study indicates that two IL-6 SNPs (rs1800796 and rs2066992) are associated with clearance of chronic HBV or protection against HBV infection at allelic, genotypic and haplotypic levels.
\end{abstract}

Correspondence to: Dr Behnaz Riazalhosseini, Department of Pharmacology, Faculty of Medicine, University of Malaya, University Street, Kuala Lumpur 50603, Malaysia

E-mail: beh.riazi@gmail.com

Abbreviations: IL-6, interleukin-6; HBV, hepatitis B virus; SNPs, single nucleotide polymorphisms; LC, liver cirrhosis; HCC, hepatocellular carcinoma; GWAS, genome wide association studies; HBsAg, HBV surface antigen; OR, odd ratio; CI, confidence interval; HWE, Hardy-Weinberg equilibrium; LD, linkage disequilibrium

Key words: interleukin-6 gene, hepatitis B virus, protective allele, polymorphism, infection resolution

\section{Introduction}

Hepatitis B virus (HBV) infection is a potentially severe disease affecting $\sim 2$ billion people worldwide $(1,2)$. It was reported by the World Health Organization in 2015 that an estimated 240 million individuals are chronically infected with the HBV virus and that 780,000 mortalities occur per year due to the consequences of $\mathrm{HBV}$ infection including liver cirrhosis (LC) and hepatocellular carcinoma (HCC) (3). The regions with high endemic rates of $\mathrm{HBV}$ infection primarily include Southeast Asia, the Western Pacific and Africa, in which incidences of more than $8 \%$ of the population have been reported (4). Thus HBV infection is a major public health issue, particularly since it exhibits variable epidemiology between different populations (4).

Numerous studies have revealed complex interactions between the host (genetic background and immune response), the virus and the environment, which may contribute to the outcomes of HBV infection (5-7). In particular, familial and twin studies have confirmed a significant role of host genetic factors in the persistence of HBV infection $(8,9)$.

Several studies, including genome wide association studies (GWAS), have revealed that single nucleotide polymorphisms (SNPs) in certain genes influence the outcomes of HBV infection (10-16). Nevertheless, there are geographical and ethnic differences between these genetic factors and the outcomes of HBV infection $(17,18)$.

Interleukin-6 (IL-6) is among the GWAS-identified genes and has been reported to be linked to susceptibility to HBV infection, clearance rate of $\mathrm{HBV}$ and also progression to severe liver disease (19-21). IL-6 is a cytokine with a vital role in the regulation of immune reactions in numerous target cells including hepatocytes (19). Previous data has indicated that host innate immunity serves a crucial role in controlling HBV infection and that $I L-6$ is a main factor involved in regulation of the innate immune response (19). It has been reported that $I L-6$ derived from activated monocytes serves a significant role in stimulating the immune response required for viral control (22). However, as a mediator of inflammation, it may also be involved in the progression of HBV-associated cirrhosis and HCC (22). Previous study documented that $I L-6$ was capable of suppressing HBV replication and inhibiting 
the accumulation of HBV covalently closed circular DNA in human hepatoma cells (23). By contrast, other studies have reported increased serum levels of $I L-6$ in patients with chronic hepatitis B (CHB) infection who developed LC/HCC. Hence, the association between $I L-6$ gene polymorphisms with susceptibility to HBV infection, clearance of HBV and progression of HBV infection is inconclusive. Therefore, the present study aimed at investigating the association between $I L-6$ gene polymorphisms (rs2069837, rs1800796 and rs2066992) and predisposition to HBV infection, clearance rate of the HBV infection and also progression of $\mathrm{HBV}$ to LC/HCC. A hospital-based case-control study was performed to examine the association among $I L-6$ SNPs and haplotypes with HBV infection risk in a Malaysian population, comprised of Malay, Chinese and Indian ethnic groups.

\section{Materials and methods}

Study participants. A total of 1,246 Malaysian subjects were recruited from the University of Malaya Medical Centre, Kuala Lumpur, Malaysia, from June 2012 to October 2014. The patient groups consisted of 423 patients with chronic $\mathrm{HBV}$ [persistence of HBV surface antigen ( $\mathrm{HBsAg}$ ) longer than 6 months] without cirrhosis or HCC and 103 patients with chronic HBV with LC and/or HCC (chronic HBV-related LC/HCC). The study cohort also consisted of $97 \mathrm{HBV}$-resolved subjects (HBsAg negative and anti-HB core antibody positive) and 623 healthy individuals (HBsAg and anti-HB core antibody negative) as controls.

LC among the chronic HBV-infected patients was confirmed by liver biopsies, or a combination of biochemical, clinical and/or radiological indications of cirrhosis (24). Diagnoses of HCC were based on radiological evidence of liver mass with arterial hypervascularity and washout in the venous-delayed phase on dynamic imaging and/or by liver histology (25). Patients with other possible causes of liver disease including primary biliary cirrhosis, Wilson's disease, hepatitis $\mathrm{C}$ infection, autoimmune hepatitis, hemochromatosis and $\alpha-1$ anti-trypsin deficiency were excluded from the study. The study protocol was approved by the Medical Ethics Committee of the University of Malaya Medical Centre (ref. no. 938.42). Ethnicities of the subjects were confirmed by a self-report. Signed and dated informed consent was obtained from all of the subjects.

Selection of SNPs. To examine whether variants in the $I L-6$ gene are capable of affecting HBV infection risk in a Malaysian population, three $I L-6$ polymorphisms ( $\mathrm{rs} 2069837 \mathrm{~A}>\mathrm{G}$, rs1800796 G>C and rs2066992 G>T) were selected based on the criteria of previous HBV studies (19-21) in other populations involving candidate genes with minor allele frequency $>0.1$ from the NCBI online database (https://www.ncbi.nlm.nih.gov).

Genotyping of IL-6 SNPs. Genomic DNA was extracted from buffy coat (isolated from $4 \mathrm{ml}$ peripheral blood following collection in anticoagulant tubes and centrifugation at $1,600 \mathrm{x}$ g for $10 \mathrm{~min}$ at room temperature) using a GeneAll ${ }^{\circledR}$ Exgene $^{\mathrm{TM}}$ DNA purification kit (GeneAll Biotechnology, Co., Ltd., Seoul, South Korea) according to the manufacturer's instructions. The 3 selected $I L-6$ polymorphisms (rs2069837, rs1800796 and rs2066992) were genotyped using a Sequenom MassARRAY ${ }^{\circledR}$ platform (Sequenom, Inc., San Diego, CA, USA) according to relevant guidelines (26). A volume of $1 \mu \mathrm{l}$ genomic DNA with a mean average concentration between $10-20 \mathrm{ng} / \mu 1$ was used in each amplification reaction. A blank and five duplicates were presented for quality control, and were excluded if any of the following occurred: assay with $>10 \%$ call rate in blank checks; $>25 \%$ call rate within the blank control; $<99.5 \%$ concordance in duplicate checks and assay with $<80 \%$ call rate in the same SpectroCHIP.

Statistical analysis. Data analysis was performed using SPSS version 16.0 (SPSS, Inc., Chicago, IL, USA). The allele and genotype distributions of the $I L-6$ SNPs between the groups were assessed by $\chi^{2}$ test. Logistic regression was performed to determine the association of $I L-6$ SNPs with (i) chronic $\mathrm{HBV}$ infection and disease progression, (ii) between chronic and resolved HBV subjects and (iii) between chronic HBV patients and healthy controls. Odd ratios (ORs) with 95\% confidence intervals (CI) were calculated following correction for confounding factors including sex and age. The SNPs were tested for deviation from Hardy-Weinberg equilibrium (HWE). Subgroup analysis based on ethnicity of allele and genotype distribution of the $I L-6$ SNPs was not performed due to the small sample size of each ethnic group.

Additionally, construction of linkage disequilibrium (LD) blocks and haplotype analysis were performed using Haploview 4.2 software (Broad Institute, Cambridge, MA, USA). Haplotype frequencies were compared between (i) chronic $\mathrm{HBV}$ cases with and without progression to LC/HCC, (ii) chronic and resolved HBV subjects and (iii) between chronic HBV patients and healthy controls, by calculating the permutation P-values created using $\chi^{2}$ statistics with one degree of freedom. $\mathrm{P}<0.05$ was considered to indicate statistical significance. The data were presented as frequency (percentage) or median (range).

\section{Results}

General characteristics of subjects. The demographics of the study participants are listed in Table I. The median ages of chronic HBV patients without LC/HCC, chronic HBV patients with LC/HCC, HBV-resolved subjects and healthy controls were 54 (range, 37-69), 66 (range, 51-79), 31 (range, 20-42) and 33 (range, 19-47), respectively.

IL-6 allele frequencies. The allelic distributions and frequencies of the $3 I L-6$ SNPs amongst chronic HBV patients, HBV-resolved subjects and healthy controls are presented in Table II. A significant difference in the allelic frequencies of $I L-6$ rs2069837, rs1800796 and rs2066992 was observed between chronic HBV patients and healthy controls $(\mathrm{OR}=0.60,95 \% \mathrm{CI}=0.47-0.78$, $\mathrm{P}<0.001$; OR=0.40, 95\% CI: 0.34-0.48, $\mathrm{P}<0.001$; and $\mathrm{OR}=0.40$, 95\% CI: 0.33-0.47, P<0.001, respectively). For rs 1800796 and rs2066992, the frequency of allele G (mutate allele) among healthy controls (0.303) was higher than that among chronic HBV patients ( 0.258 for rs1800796 and 0.265 for rs2066992). There were no significant differences in allelic frequencies for rs2069837, rs1800796 and rs2066992 between chronic HBV cases with and without LC/HCC or between chronic HBV 


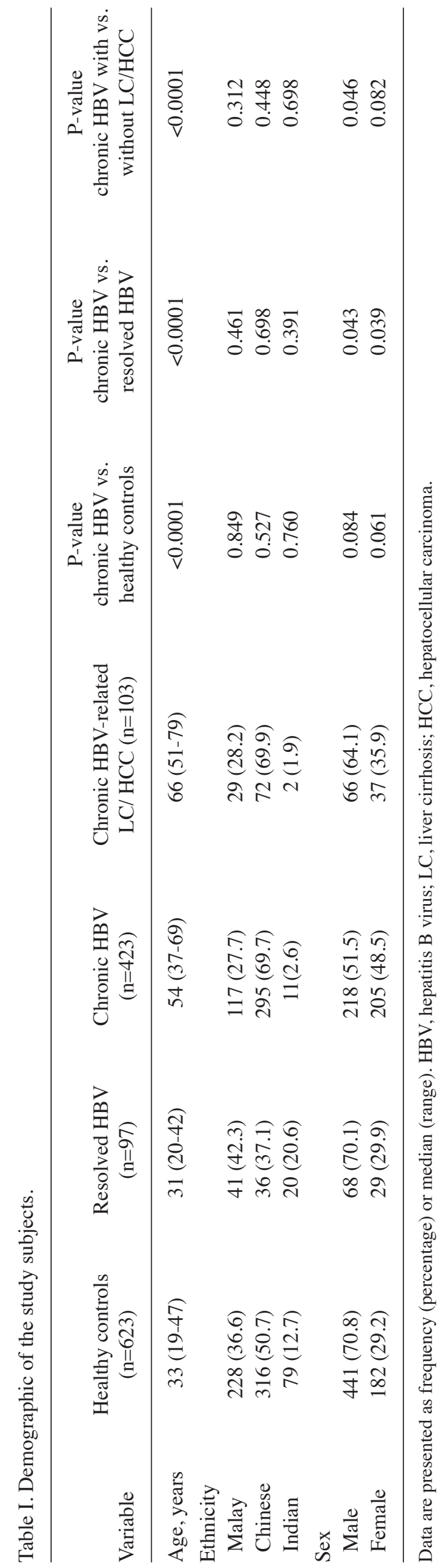

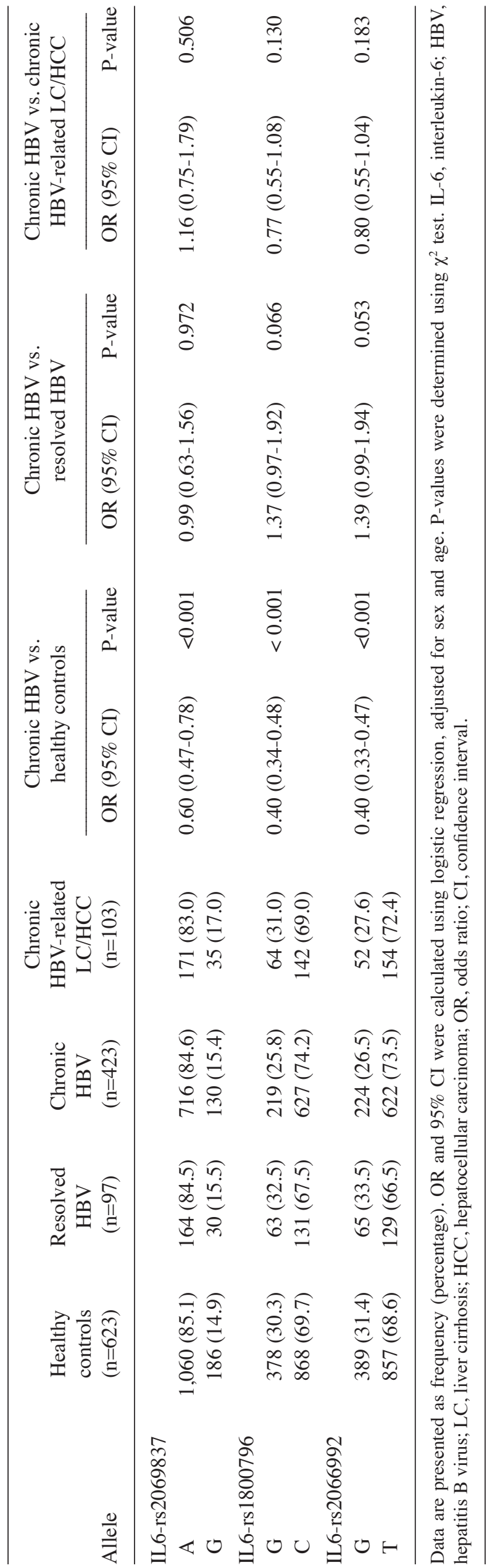




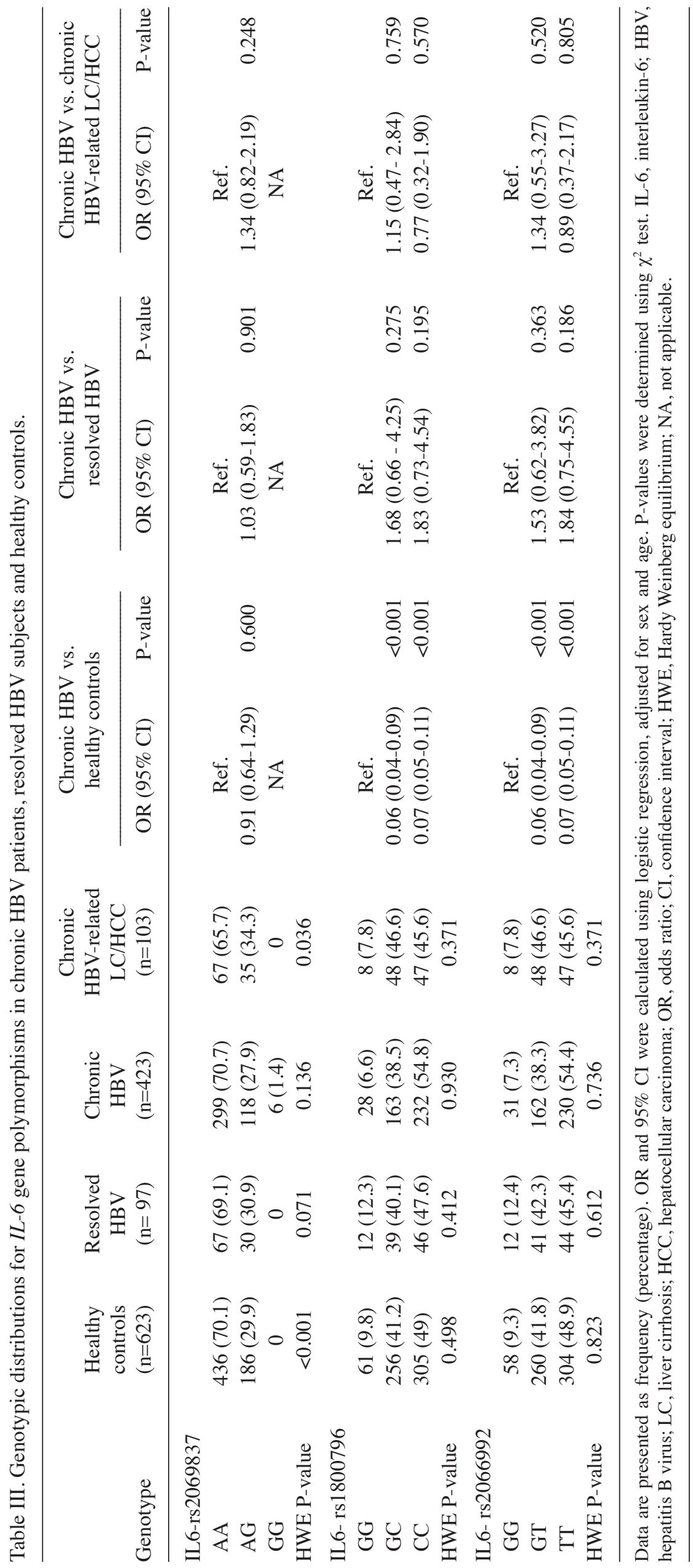


Table IV. Haplotype analysis of IL-6 rs1800796 and rs2066992 association between chronic HBV patients, healthy controls and HBV-resolved subjects.

\begin{tabular}{|c|c|c|c|c|c|c|c|}
\hline \multirow[b]{2}{*}{ Comparison } & \multirow[b]{2}{*}{ Haplotype } & \multicolumn{6}{|c|}{ Frequency } \\
\hline & & All & Case $^{\mathrm{a}}$ & Control $^{\mathrm{b}}$ & $\chi^{2}$ & P-value & $\mathrm{D}^{\prime}$ \\
\hline \multirow[t]{3}{*}{ Chronic HBV vs. healthy controls } & Block 1 & & & & & & 0.98 \\
\hline & CT & 0.709 & 0.745 & 0.688 & 7.727 & 0.005 & \\
\hline & GG & 0.282 & 0.245 & 0.304 & 8.423 & 0.003 & \\
\hline \multirow[t]{3}{*}{ Chronic HBV vs. resolved HBV } & Block 1 & & & & & & 0.99 \\
\hline & $\mathrm{CT}$ & 0.720 & 0.752 & 0.694 & 4.309 & 0.037 & \\
\hline & GG & 0.269 & 0.237 & 0.295 & 4.443 & 0.035 & \\
\hline \multirow[t]{3}{*}{ Chronic HBV vs. chronic HBV- related LC/HCC } & Block 1 & & & & & & 0.99 \\
\hline & CT & 0.724 & 0.689 & 0.733 & 0.129 & 2.312 & \\
\hline & GG & 0.266 & 0.311 & 0.255 & 0.114 & 2.108 & \\
\hline
\end{tabular}

${ }^{\mathrm{a} C a s e}$ for comparison 1 is chronic HBV patients, for comparison 2 is chronic HBV patients, for comparison 3 is HBV-related LC/HCC; ${ }^{\mathrm{b}}$ control for comparison 1 is healthy controls, for comparison 2 is resolved HBV subjects and for comparison 3 is chronic HBV. IL-6, interleukin-6; HBV, hepatitis B virus; LC, liver cirrhosis; HCC, hepatocellular carcinoma.

patients and HBV-resolved subjects; although association was indicated for IL-6 rs2066992 between chronic HBV patients and HBV-resolved HBV subjects $(\mathrm{P}=0.053)$.

IL-6 genotype frequencies. The genotypic distributions of the 3 IL-6 SNPs among chronic HBV patients, HBV-resolved subjects and healthy controls are presented in Table III. A significant difference in the genotype frequencies of $I L-6$ rs1800796 was identified between chronic HBV patients and healthy controls $(\mathrm{OR}=0.06,95 \% \mathrm{CI}: 0.04-0.09, \mathrm{P}<0.001$ for $\mathrm{GC}$ genotype and $\mathrm{OR}=0.07,95 \% \mathrm{CI}$ : $0.05-0.11, \mathrm{P}<0.001$ for CC genotype). Similarly, a significant association was observed in genotype frequencies of $I L-6$ rs2066992 between chronic HBV patients and healthy controls $(\mathrm{OR}=0.06$; 95\% CI: $0.04-0.09, \mathrm{P}<0.001$ for GT genotype and $\mathrm{OR}=0.07$, 95\% CI: $0.05-0.11, \mathrm{P}<0.001$ for TT genotype). There were no significant differences in the genotypic frequencies of rs2069837, rs1800796 and rs2066992 between chronic HBV cases with and without LC/HCC or between chronic HBV patients and HBV-resolved subjects. The genotype distribution of rs2069837, rs1800796 and rs2066992 were in HWE (P>0.05 for each) except for rs2069837 in chronic HBV-related LC/HCC cases $(\mathrm{P}=0.036)$ and the healthy control group $(\mathrm{P}<0.001)$.

IL-6 haplotype frequencies. The results of three haplotype analyses performed between (i) chronic HBV cases with and without LC/HCC, (ii) chronic HBV patients and HBV-resolved subjects and (iii) chronic HBV patients and healthy controls are presented in Table IV. The haplotype combination, CT and GG, was generated between IL6 rs1800796 and rs2066992. IL6 rs2069837 was excluded from analysis due to deviation from HWE. Significant associations with strong LD $\left(D^{\prime}=0.98\right)$ was observed of haplotypes CT and GG in the comparison of chronic HBV patients vs. healthy controls $(\mathrm{P}=0.005$, frequency 0.709 and $\mathrm{P}=0.003$, frequency 0.282 , respectively). Similarly, the haplotype results on comparing the chronic $\mathrm{HBV}$ and $\mathrm{HBV}$-resolved groups indicated significant association with strong LD ( $\left.\mathrm{D}^{\prime}=0.99\right)$.
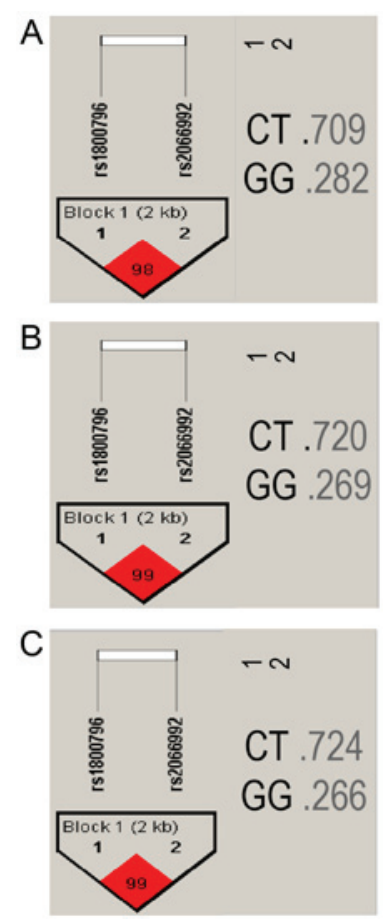

Figure 1. LD analysis of two IL-6 SNPs (rs1800796 and rs2066992) in three comparisons. (A) Pairwise LD between chronic HBV patients and healthy controls. (B) Pairwise LD between chronic HBV patients and HBV-resolved subjects. (C) Pairwise LD between chronic HBV patients with and without cirrhosis/HCC. LD, linkage disequilibrium; IL-6, interleukin-6; SNPs, single nucleotide polymorphisms; HBV, hepatitis B virus; HCC, hepatocellular carcinoma.

Haplotype CT $(\mathrm{P}=0.037$, frequency 0.720$)$ and haplotype $\mathrm{GG}$ $(\mathrm{P}=0.035$, frequency 0.269$)$ were significantly associated with clearance of the HBV virus. Although the IL6 rs1800796 and rs2066992 SNPs between chronic HBV cases with and without $\mathrm{LC} / \mathrm{HCC}$ were in strong $\mathrm{LD}\left(\mathrm{D}^{\prime}=0.99\right)$, there were no significant associations with progression of chronic HBV infection. The LD pattern in the three comparisons is depicted in Fig. 1. 


\section{Discussion}

In the present study, two SNPs (rs1800796 and rs2066992) in the $I L-6$ gene were identified to be associated with protection against HBV infection in a Malaysian population. It was observed that the G allele in both SNPs (rs1800796 and rs2066992) may have a significant protective role against HBV infection. The results also indicated that the GG and CT haplotypes of IL6 rs1800796 and rs2066992 were associated with protection against HBV infection (in comparison with healthy controls) as well as with clearance of HBV (in comparison with HBV-resolved subjects). For $I L-6$ rs1800796, the genotypes GG and GC containing the protective allele G were more frequently identified among healthy controls as compared with chronic HBV patients. Saxena et al (27) similarly reported that the GG genotype of $I L-6$ rs1800796 was more frequent in healthy controls as compared with chronic HBV patients. However, for $I L-6$ rs2066992, genotypes GG and GT containing the protective allele $\mathrm{G}$ were predominant among healthy controls as compared with chronic HBV patients (27).

There was no significant difference between the ethnicity and the study subjects. Nonetheless, the study had higher number of Chinese participants compared to Malays and Indian. This is probably due to the fact that CHB infection is generally common among Chinese in Malaysia (28)

Host genetic background and its interaction with HBV may affect the outcomes of long-term infection (29). IL- 6 has been demonstrated to be involved in HBV infection in vitro and in vivo (30). Emerging data has revealed that the $I L-6$ polymorphism rs1800796 may be associated with differences in the outcomes of hepatitis B infection including progression to $\mathrm{LC} / \mathrm{HCC}$ as well as clearance of the virus $(19,20)$. It is speculated that genetic alteration in the $I L-6$ gene has an effect on immune dysfunction, which may be involved in the pathogenesis of HBV infection (31). It has been reported that variation in the $I L-6$ gene resulting in functional alteration, which may be responsible for changes in the levels of transcription, protein expression and serum $I L-6$, were correlated with HBV disease outcomes (22). Furthermore, it has been indicated that $I L-6$ contributes to the interaction between the hepatocyte plasma membrane and the HBV particle (32); HBV could then bind to the preS1 domain on human hepatocytes, mediating HBV infection (31). As a result of activating these signaling pathways, $I L-6$ may increase the activity of the HBV enhancer 1 to control the expression of HBV X protein and the replication of HBV (32). A study by Chou et al (33) reported that $I L-6$ in hepatoma cells promoted HBV transcription by activating signal transducer and activator of transcription 3 , which may interact with hepatocyte nuclear factor 3 bound to the HBV enhancer.

In the present study, the rs1800796-G allele (mutant allele) was identified to be the minor allele compared with the $\mathrm{C}$ allele (wild-type allele) among healthy controls. A similar result was observed for rs2066992-G allele (mutant allele) as compared with the T allele (wild-type allele) in the same group. In addition, it was identified that rs1800796 and rs2066992 genotypes were significantly associated with protection from HBV infection. However, no association was observed on comparison between chronic HBV and HBV-resolved subjects or between chronic
$\mathrm{HBV}$ cases with and without $\mathrm{LC} / \mathrm{HCC}$. This is contradictory to a study by Lu et al (19), which reported that genetic variation in the SNP IL-6-572 C/G may be associated with clearance of HBV infection. Furthermore, the results of another study revealed that genetic variation in the SNP $I L-6-572$ may be associated with HCC incidence in males (34).

In the present study no significant difference was identified in the genotype/allele frequencies of rs2069837, rs1800796 and rs2066992 between chronic HBV patients with and without LC/HCC. Hence, these SNPs are likely not associated with progression to liver disease in $\mathrm{HBV}$-infected patients in the Malaysian population. Furthermore, it was observed that these $I L-6$ variants were not associated with clearance of $\mathrm{HBV}$ infection. Similarly, Park et al (35) reported that $I L-6$ variants were not associated with $\mathrm{HBV}$ infection or the progression of HBV-associated liver disease.

Considering that haplotypes may be more effective markers of association regarding disease-linked genes than SNPs (36), the association of $I L-6$ haplotypes with progression and clearance of HBV infection was examined. All three SNPs are common variants including the functional polymorphism of the $I L-6$ promoter (rs1800796) and the SNPs in the intron 2 region (rs2069837 and rs2066992) (37). Significant associations were observed for haplotypes GG and CT on comparison of individuals with chronic HBV with healthy controls and HBV-resolved subjects. Notably, the haplotype results indicated that the wild-type haplotype (GG) had higher frequency in healthy controls as compared with chronic HBV patients. Similarly, the haplotype GG had higher frequency in HBV-resolved patients as compared with chronic HBV patients. However, the haplotype CT exhibited a significant association with chronic HBV as compared with healthy controls and HBV-resolved subjects, with the highest frequency observed in chronic HBV patients.

The present study to the best of our knowledge is the first to investigate the association among $I L-6$ SNPs and HBV infection risk in a Malaysian population. Nonetheless, the present study had limitations with regard to functional insights into the role of $I L-6$ in HBV infection. Thus, functional studies using mammalian cell lines are required in order to investigate the transcriptional activities of the $I L-6$ SNPs.

In conclusion, association was identified between polymorphic variants of the wild-type alleles of rs1800796 and rs2066992 of $I L-6$ and protection from HBV infection among a Malaysian population. Similarly, Lu et al reported that allele $\mathrm{G}$ was associated with the natural elimination of HBV infection (19). Furthermore, study has indicated that the $\mathrm{G}$ allele is associated with significantly elevated circulating levels of IL-6 (27). These findings are consistent with current results and support the apparent protective role of allele $\mathrm{G}$ in HBV infection. It was also indicated that haplotypes GG and CT of $I L-6$ may have a significant protective role with regard to hepatitis B disease outcomes in Malaysian individuals. However, functional studies and more diverse cohorts are required to provide wider insight into the role of $I L-6$ in HBV infection.

\section{Acknowledgements}

Not applicable. 


\section{Funding}

The present study was supported by the High Impact Research Ministry of Higher Education Malaysia (HIR MOHE; grant no. H-20001-00-E000063) and by a University of Malaya postgraduate research grant (grant no. PG146-2014B). The funders had no role in study design, data collection or analysis, decision to publish, or preparation of the manuscript.

\section{Availability of data and materials}

All data generated or analysed during this study are included in this published article.

\section{Authors' contributions}

BR, ZM and RM conceived and designed the study. BR and NSS performed sample collection and DNA extraction. BR performed the assays, analysed the data and wrote the manuscript. ZM, RM and YDA provided critical revisions on the article. All authors read and approved the final manuscript.

\section{Ethics approval and consent to participate}

The present study was approved by the Medical Ethics Committee of the University of Malaya Medical Centre, Kuala Lumpur, Malaysia (ref. no. 938.42). Signed and dated informed consent was obtained from all of the subjects prior to their participation in the study.

\section{Patient consent for publication}

Written informed consent was obtained from each participant agreeing to the publication of their data on the terms of data anonymization.

\section{Competing interests}

The authors declare that they have no competing interests.

\section{References}

1. Tang S, Liu Z, Zhang Y, He Y, Pan D, Liu Y, Liu Q, Zhang Z and Yuan Y: Rather than Rs1800796 polymorphism, expression of interleukin-6 is associated with disease progression of chronic HBV infection in a Chinese Han population. Dis Markers 35: 799-805, 2013

2. Zhang P, Li N, Zhu Q, Li F, Yang C, Zeng X, Lv Y, Zhou Z, Han Q and Liu Z: Association between TNFAIP3 nonsynonymous single-nucleotide polymorphism rs2230926 and chronic hepatitis $\mathrm{B}$ virus infection in a Chinese Han population. Virol J 12: 33, 2015.

3. World Health Organization (WHO): Hepatitis B. Fact sheet No 204. WHO, Geneva, 2015. http://www.who.int/mediacentre/ factsheets/fs204_Jul2014/en/. Updated July, 2015.

4. Papastergiou V, Lombardi R, MacDonald D and Tsochatzis EA: Global Epidemiology of Hepatitis B Virus (HBV) Infection. Curr Hepatol Rep 14: 171-178, 2015.

5. Zeng Z: Human genes involved in hepatitis B virus infection. World J Gastroenterol 20: 7696-7706, 2014.

6. Araujo NM, Waizbort R and Kay A: Hepatitis B virus infection from an evolutionary point of view: How viral, host, and environmental factors shape genotypes and subgenotypes. Infect Genet Evol 11: 1199-1207, 2011.

7. Ali A, Abdel-Hafiz H, Suhail M, Al-Mars A, Zakaria MK, Fatima K, Ahmad S, Azhar E, Chaudhary A and Qadri I: Hepatitis B virus, HBx mutants and their role in hepatocellular carcinoma. World J Gastroenterol 20: 10238-10248, 2014.
8. He YL, Zhao YR, Zhang SL and Lin SM: Host susceptibility to persistent hepatitis B virus infection. World J Gastroenterol 12: 4788-4793, 2006.

9. Guo X, Zhang Y, Li J, Ma J, Wei Z, Tan W and O'Brien SJ: Strong influence of human leukocyte antigen (HLA)-DP gene variants on development of persistent chronic hepatitis B virus carriers in the Han Chinese population. Hepatology 53: 422-428, 2011

10. Mbarek H, Ochi H, Urabe Y, Kumar V, Kubo M, Hosono N, Takahashi A, Kamatani Y, Miki D, Abe H, et al: A genome-wide association study of chronic hepatitis B identified novel risk locus in a Japanese population. Hum Mol Genet 20: 3884-3892, 2011.

11. Al-Qahtani A, Khalak HG, Alkuraya FS, Al-hamoudi W, Alswat K, Al Balwi MA, Al Abdulkareem I, Sanai FM and Abdo AA: Genome-wide association study of chronic hepatitis B virus infection reveals a novel candidate risk allele on 11q22.3. J Med Genet 50: 725-732, 2013.

12. Hu Z, Liu Y, Zhai X, Dai J, Jin G, Wang L, Zhu L, Yang Y, Liu J, Chu M, et al: New loci associated with chronic hepatitis B virus infection in Han Chinese. Nat Genet 45: 1499-1503, 2013.

13. Jiang DK, Sun J, Cao G, Liu Y, Lin D, Gao YZ, Ren WH, Long XD, Zhang H, Ma XP, et al: Genetic variants in STAT4 and HLA-DQ genes confer risk of hepatitis B virus-related hepatocellular carcinoma. Nat Genet 45: 72-75, 2013.

14. Li S, Qian J, Yang Y, Zhao W, Dai J, Bei JX, Foo JN, McLaren PJ, Li Z, Yang J, et al: GWAS identifies novel susceptibility loci on 6 p21.32 and 21q21.3 for hepatocellular carcinoma in chronic hepatitis B virus carriers. PLoS Genet 8: e1002791, 2012.

15. Kim YJ, Kim HY, Lee JH, Yu SJ, Yoon JH, Lee HS, Kim CY, Cheong JY, Cho SW, Park NH, et al: A genome-wide association study identified new variants associated with the risk of chronic hepatitis B. Hum Mol Genet 22: 4233-4238, 2013.

16. Nishida N, Sawai H, Matsuura K, Sugiyama M, Ahn SH, Park JY, Hige S, Kang JH, Suzuki K, Kurosaki M, et al: Genome-wide association study confirming association of HLA-DP with protection against chronic hepatitis B and viral clearance in Japanese and Korean. PLoS One 7: e39175, 2012.

17. El-Serag HB: Hepatocellular carcinoma. N Engl J Med 365: 1118-1127, 2011.

18. Karlsen TH, Melum E and Franke A: The utility of genome-wide association studies in hepatology. Hepatology 51: 1833-1842, 2010.

19. Lu Y, Peng J, Wang C, Zhu Y, Wang F and Sun Z: IL-6 promoter functional polymorphism $-572 \mathrm{C} / \mathrm{G}$ affects spontaneous clearance of hepatitis B virus infection. Clin Lab 60: 1903-1907, 2014.

20. Tang S, Yuan Y, He Y, Pan D, Zhang Y, Liu Y, Liu Q, Zhang Z and Liu Z: Genetic polymorphism of interleukin-6 influences susceptibility to HBV-related hepatocellular carcinoma in a male Chinese Han population. Hum Immunol 75: 297-301, 2014.

21. Zhao X-M, Gao Y-F, Zhou Q, Pan F-M and Li X: Relationship between interleukin- 6 polymorphism and susceptibility to chronic hepatitis B virus infection. World J Gastroenterol 19: 6888-6893, 2013

22. Lan T, Chang L, Wu L and Yuan Y-F: IL-6 plays a crucial role in HBV infection. J Clin Transl Hepatol 3: 271-276, 2015.

23. Kuo TM, Hu CP, Chen YL, Hong MH, Jeng KS, Liang CC, Chen ML and Chang C: HBV replication is significantly reduced by IL-6. J Biomed Sci 16: 41, 2009.

24. Chook JB, Ngeow YF, Yap SF, Tan TC and Mohamed R: Combined use of wild-type HBV precore and high serum iron marker as a potential tool for the prediction of cirrhosis in chronic hepatitis B infection. J Med Virol 83: 594-601, 2011.

25. Bruix J and Sherman M; American Association for the Study of Liver Diseases: Management of hepatocellular carcinoma: An update. Hepatology 53: 1020-1022, 2011.

26. Bradić M, Costa J and Chelo IM: Genotyping with Sequenom. Methods Mol Biol 772: 193-210, 2011.

27. Saxena R, Chawla YK, Verma I and Kaur J: IL-6(-572/-597) polymorphism and expression in HBV disease chronicity in an Indian population. Am J Hum Biol 26: 549-555, 2014.

28. Yap SF: Chronic hepatitis B infection in Malaysians. Malays J Pathol 16: 3-6, 1994.

29. Tunçbilek $\mathrm{S}$ : Relationship between cytokine gene polymorphisms and chronic hepatitis B virus infection. World J Gastroenterol 20: 6226-6235, 2014

30. Galun E, Nahor O, Eid A, Jurim O, Rose-John S, Blum HE, Nussbaum O, Ilan E, Daudi N, Shouval D, et al: Human interleukin- 6 facilitates hepatitis B virus infection in vitro and in vivo. Virology 270: 299-309, 2000. 
31. De Meyer S, Gong ZJ, Suwandhi W, van Pelt J, Soumillion A and Yap SH: Organ and species specificity of hepatitis B virus (HBV) infection: A review of literature with a special reference to preferential attachment of HBV to human hepatocytes. J Viral Hepat 4: 145-153, 1997.

32. Ohno H, Kaneko S, Kobayashi K and Murakami S: Human hepatitis B virus enhancer 1 is responsive to human interleukin-6. J Med Virol 52: 413-418, 1997.

33. Chou CH, Chen PJ, Jeng YM, Cheng AL, Huang LR and Cheng JC: Synergistic effect of radiation and interleukin- 6 on hepatitis B virus reactivation in liver through STAT3 signaling pathway. Int J Radiat Oncol Biol Phys 75: 1545-1552, 2009.

34. Liu S, Qiu XQ, Zeng XY, Bai $\mathrm{H}$, Bei $\mathrm{CH}$ and Yang $\mathrm{Y}$ : Relationship between IL6 -572G/C polymorphism and hepatocellular carcinoma in men. Zhonghua Gan Zang Bing Za Zhi 20: 463-467, 2012 (In Chinese).
35. Park BL, Lee HS, Kim YJ, Kim JY, Jung JH, Kim LH and Shin HD: Association between interleukin 6 promoter variants and chronic hepatitis B progression. Exp Mol Med 35: 76-82, 2003.

36. Manolio TA, Brooks LD and Collins FS: A HapMap harvest of insights into the genetics of common disease. J Clin Invest 118: 1590-1605, 2008.

37. Chen J, Liu RY, Yang L, Zhao J, Zhao X, Lu D, Yi N, Han B, Chen XF, Zhang K, et al: A two-SNP IL-6 promoter haplotype is associated with increased lung cancer risk. J Cancer Res Clin Oncol 139: 231-242, 2013. 